\title{
Article \\ Profiling of Adipose and Skeletal Muscle in Human Pancreatic Cancer Cachexia Reveals Distinct Gene Profiles with Convergent Pathways
}

\author{
Ashok Narasimhan ${ }^{1}$, Xiaoling Zhong ${ }^{1,2}$, Ernie P. Au ${ }^{1,3,+}$, Eugene P. Ceppa ${ }^{1}$, Atilla Nakeeb ${ }^{1}$, \\ Michael G. House ${ }^{1,2,4}$, Nicholas J. Zyromski ${ }^{1}$, C. Max Schmidt ${ }^{1}$, Katheryn N. H. Schloss ${ }^{1, \ddagger}$, Daniel E. I. Schloss ${ }^{1, \S}$, \\ Yunlong Liu 2,4,5,6,7 D, Guanglong Jiang ${ }^{5}$, Bradley A. Hancock ${ }^{1}$, Milan Radovich 1,4, Joshua K. Kays ${ }^{1}$, \\ Safi Shahda $2,4,8$, Marion E. Couch $2,4,9, \|$, Leonidas G. Koniaris ${ }^{1,2,4,7}$ and Teresa A. Zimmers 1,2,3,4,6,7,9,10,*(D)
}

check for updates

Citation: Narasimhan, A.; Zhong, X.; Au, E.P.; Ceppa, E.P.; Nakeeb, A.; House, M.G.; Zyromski, N.J.; Schmidt, C.M.; Schloss, K.N.H.; Schloss, D.E.I.; et al. Profiling of Adipose and Skeletal Muscle in Human Pancreatic Cancer Cachexia Reveals Distinct Gene Profiles with Convergent Pathways. Cancers 2021, 13, 1975. https://doi.org/10.3390/cancers13081975

Academic Editor: Rolf A. Brekken

Received: 25 March 2021

Accepted: 14 April 2021

Published: 20 April 2021

Publisher's Note: MDPI stays neutral with regard to jurisdictional claims in published maps and institutional affiliations.

Copyright: (c) 2021 by the authors. Licensee MDPI, Basel, Switzerland. This article is an open access article distributed under the terms and conditions of the Creative Commons Attribution (CC BY) license (https:/ / creativecommons.org/licenses/by/ $4.0 /)$.
1 Department of Surgery, Indiana University School of Medicine, Indianapolis, IN 46202, USA; ashnaras@iu.edu (A.N.); xzhong@iu.edu (X.Z.); au.ernie@gmail.com (E.P.A.); eceppa@iupui.edu (E.P.C.); anakeeb@iupui.edu (A.N.); michouse@iupui.edu (M.G.H.); nzyromsk@iupui.edu (N.J.Z.); maxschmi@iupui.edu (C.M.S.); schlosskn@upmc.edu (K.N.H.S.); schlossde@upmc.edu (D.E.I.S.); hancockb@iupui.edu (B.A.H.); mradovic@iupui.edu (M.R.); joshuakays@gmail.com (J.K.K.); lkoniari@iu.edu (L.G.K.)

2 IUPUI Center for Cachexia Research Innovation and Therapy, Indianapolis, IN 46202, USA; yunliu@iu.edu (Y.L.); safi.shahda@lilly.com (S.S.); marioncouch444@gmail.com (M.E.C.)

3 Department of Biochemistry and Molecular Biology, Indiana University School of Medicine, Indianapolis, IN 46202, USA

4 Indiana University Simon Cancer Center, Indianapolis, IN 46202, USA

5 Department of Medical and Molecular Genetics, Indiana University School of Medicine, Indianapolis, IN 46202, USA; ggjiang@iu.edu

6 Center for Computational Biology and Bioinformatics, Indiana University School of Medicine, Indianapolis, IN 46202, USA

Indiana Center for Musculoskeletal Health, Indianapolis, IN 46202, USA

8 Department of Medicine, Indiana University School of Medicine, Indianapolis, IN 46202, USA

9 Department of Otolaryngology-Head \& Neck Surgery, Indiana University School of Medicine, Indianapolis, IN 46202, USA

10 Department of Anatomy, Cell Biology \& Physiology, Indiana University School of Medicine, Indianapolis, IN 46202, USA

* Correspondence: zimmerst@iu.edu; Tel.: +1-(317)-278-7289

+ Current Address: Berkeley Lights, Emeryville, CA 94608, USA.

$\ddagger$ Current Address: UPMC Children's Hospital of Pittsburgh, Pittsburgh, PA 15224, USA.

$\S$ Current Address: Department of Emergency Medicine, University of Pittsburgh School of Medicine, Pittsburgh, PA 15213, USA.

|| Current Address: Cambia Health Solutions, OR 97201, USA.

Simple Summary: More than $80 \%$ of patients with pancreatic ductal adenocarcinoma (PDAC) suffer cachexia, characterized by loss of muscle and fat. However, most cachexia studies were predominantly focused on muscle. Our clinical study showed adipose tissue loss as a prognosticator in PDAC cachexia. Our study aims to understand the concurrent muscle and adipose changes using transcriptome profiling. We identified tissue-specific gene expression profiles with changes in adipose being more dynamic. Pathway analysis suggests that muscle and adipose wasting may be mediated through independently targetable mechanisms which may have therapeutic implications. Many of the well-known and novel cachexia genes have been validated using an external muscle and adipose datasets. The study provides the groundwork for future studies to understand if fat wasting precedes muscle wasting in PDAC and if adipose can be targeted for therapeutic interventions. The study also shows that age related muscle loss has distinct mechanisms compared to cachexia.

Abstract: The vast majority of patients with pancreatic ductal adenocarcinoma (PDAC) suffer cachexia. Although cachexia results from concurrent loss of adipose and muscle tissue, most studies focus on muscle alone. Emerging data demonstrate the prognostic value of fat loss in cachexia. Here we sought to identify the muscle and adipose gene profiles and pathways regulated in cachexia. Matched rectus abdominis muscle and subcutaneous adipose tissue were obtained at surgery from 
patients with benign conditions $(n=11)$ and patients with PDAC $(n=24)$. Self-reported weight loss and body composition measurements defined cachexia status. Gene profiling was done using ion proton sequencing. Results were queried against external datasets for validation. $961 \mathrm{DE}$ genes were identified from muscle and 2000 from adipose tissue, demonstrating greater response of adipose than muscle. In addition to known cachexia genes such as FOXO1, novel genes from muscle, including PPP1R8 and AEN correlated with cancer weight loss. All the adipose correlated genes including SCGN and EDR17 are novel for PDAC cachexia. Pathway analysis demonstrated shared pathways but largely non-overlapping genes in both tissues. Age related muscle loss predominantly had a distinct gene profiles compared to cachexia. This analysis of matched, externally validate gene expression points to novel targets in cachexia.

Keywords: pancreatic cancer; pancreatic ductal adenocarcinoma; gene expression; RNAseq; adipose; skeletal muscle; atrophy; cachexia; neoplasia

\section{Introduction}

Cancer associated cachexia is a debilitating multifactorial syndrome characterized by involuntary loss of muscle and fat [1-4]. Patients with pancreatic ductal adenocarcinoma (PDAC), the major subtype of pancreatic cancer, have a higher proclivity to develop cachexia; such cachexia is significantly associated with PDAC-related deaths [5-8]. The pathophysiology of PDAC cachexia involves a complex interplay between the host and tumor interactions which results in inflammation, malnutrition, anorexia, and neuroendocrine changes. These complex interactions lead to a series of metabolic changes including Warburg effect resulting in increased Cori cycle, lipolysis and decreased lipogenesis [9-11]. Studies using animal models have helped uncover many mechanisms that cause muscle and fat wasting [12-18], and newer animal models are constantly developed to study PDAC and understand cachexia mechanisms $[19,20]$. Although most of our understanding about cachexia comes from muscle, it is known that cachexia often involves wasting of fat $[4,21-24]$. Fat wasting is prognostic in PDAC cachexia. In one cohort study, patients with PDAC who lost only fat had similarly reduced survival to those with combined fat and muscle loss (10 months less) relative to patients who lose neither, independent of tumor response to therapy [4]. However, the molecular mechanisms of fat wasting in PDAC cachexia are less explored.

Understanding the human biology of cancer cachexia is challenging and datasets to date have been limited. Most human cachexia studies to date have used muscle and fat from multiple cancer types [25]. However, though certain genes may be common across cancer types, it seems likely that there will be both tumor-type and treatment-specific mechanisms. Studying a single cancer type could reduce the heterogeneity and begin to interrogate cachexia in a cancer-specific manner. In human studies as well, the focus has either been muscle or fat but to date, never both.

Our current study aims to address several of these challenges in human PDAC cachexia. The aims of the study are to (i) identify differentially expressed (DE) genes for muscle and adipose from the same individuals, (ii) identify common and unique pathways between muscle and fat in cachexia, (iii) identify tissue specific transcription factors, cytokines, growth factors and receptors, (iv) correlate all profiled genes from muscle and fat to the clinical variable of cancer weight loss grade (CWLG) [2] (v) to validate the differentially expressed (DE) muscle and adipose genes in external datasets and (vi) understand if age related muscle loss has distinct gene signatures when compared to PDAC cachexia. Our study identified differentially expressed, distinct tissue-specific gene expression signatures. Genes within common pathways were differentially present in muscle and adipose. We found genes commonly identified in animal models of cachexia, such as FOXO1, FOXO3, IL6R, ZIP14 and PIK3R1, manifested in two independent muscle human datasets. Inflammation-related pathways emerged as key component from the 
adipose tissue validation data. Our results also suggest that age related muscle loss had a predominantly distinct gene signatures and regulated pathways when compared to PDAC cachexia indicating that age related muscle loss has distinct molecular mechanisms compared to PDAC cachexia.

This is the first study to perform cachexia-specific muscle and adipose gene profiling from same subjects, the first to report genes associated with fat wasting in PDAC cachexia, and the first to validate both findings in external dataset. We demonstrate that muscle and adipose transcriptomes are well established early in pancreatic cancer and that the adipose response is profound than the muscle response. These distinct, tissue-specific gene expression profiles and pathway modulation suggests that muscle and adipose wasting in cachexia may be mediated through independently targetable mechanisms.

\section{Results}

\subsection{Study Design and Patient Demographics}

The overall study design is shown in Figure 1. The summary of patient demographics is presented in Table 1 . The mean age of controls and PDAC was different, $50 \pm 14$ versus $70 \pm 11$ years $(p=0.001)$. While sex and BMI were not different between groups, weight loss grade was increased in patients versus controls $(p=0.005)$. By CT analysis, skeletal muscle index was significantly less in PDAC patients, while total adipose tissue index was not. However, intramuscular fat was significant between groups and subcutaneous adipose tissue was trending towards significance (Table 1).

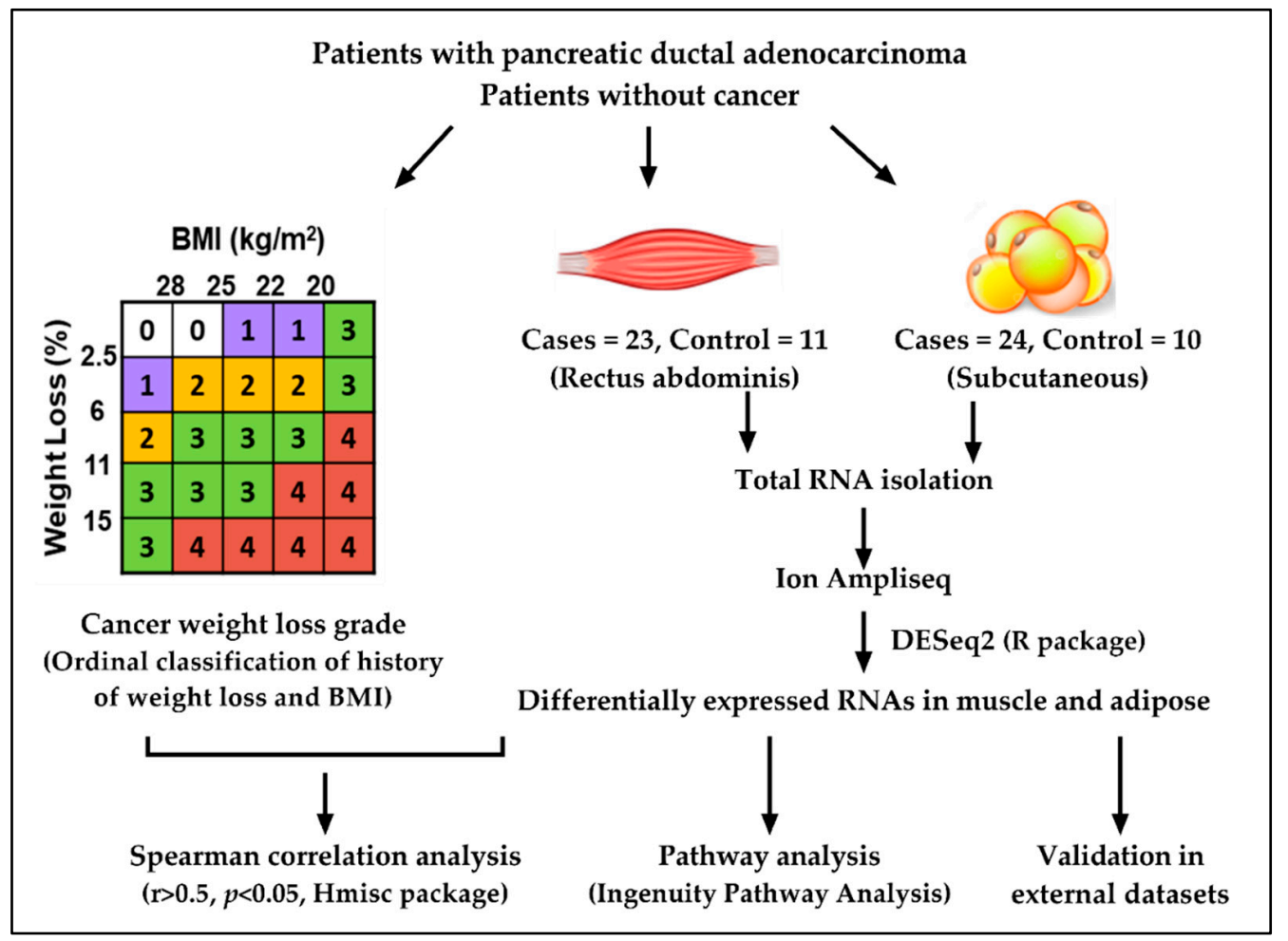

Figure 1. Overall study design. 
Table 1. Patient demographics.

\begin{tabular}{|c|c|c|c|}
\hline Characteristics & $\begin{array}{c}\text { PDAC } \\
n=24\end{array}$ & $\begin{array}{c}\text { Non-Cancer Controls } \\
\qquad n=11\end{array}$ & $p$-Value \\
\hline Age $^{a}$ & $70 \pm 11$ & $50 \pm 14$ & 0.001 \\
\hline \multicolumn{4}{|l|}{ Gender ${ }^{b}$} \\
\hline Male & 12 & 5 & \multirow[t]{2}{*}{ NS } \\
\hline Female & 12 & 6 & \\
\hline $\operatorname{BMI}\left(\mathrm{kg} / \mathrm{m}^{2}\right)^{\mathrm{a}}$ & $28.2 \pm 6.5$ & $31.4 \pm 6$ & NS \\
\hline \multicolumn{4}{|l|}{ Weight Loss Grade ${ }^{c}$} \\
\hline Grade 0 & 3 & 6 & \multirow{5}{*}{0.005} \\
\hline Grade 1 & 1 & 3 & \\
\hline Grade 2 & 6 & 1 & \\
\hline Grade 3 & 8 & 1 & \\
\hline Grade 4 & 6 & - & \\
\hline Skeletal muscle index $\left(\mathrm{cm}^{2} / \mathrm{m}^{2}\right)^{a}$ & $44.6 \pm 10.5$ & $53.2 \pm 9.7$ & 0.04 \\
\hline Total adipose index $\left(\mathrm{cm}^{2} / \mathrm{m}^{2}\right)^{a}$ & $235.2 \pm 134.2$ & $274 \pm 150.8$ & NS \\
\hline Intramuscular Fat $\left(\mathrm{cm}^{2}\right)$ & $17.65 \pm 13.46$ & $10.58 \pm 3.90$ & 0.03 \\
\hline Subcutaneous Fat $\left(\mathrm{cm}^{2}\right)$ & $226.07 \pm 119.09$ & $316.97 \pm 110.97$ & 0.055 \\
\hline
\end{tabular}

$\mathrm{N} \mathrm{S}=$ Not Significant; $^{\mathrm{a}}=$ t-test, $^{\mathrm{b}}=$ Chi-square test, ${ }^{\mathrm{c}}=$ Fisher's exact test. The values are represented as mean \pm standard deviation. $p<0.05$ were considered statistically significant.

\subsection{Muscle and Adipose Tissue Have Distinct Gene Expression Patterns}

In all, 14,177 genes were profiled in muscle and 12,910 genes were profiled in adipose tissue. All the profiled genes served as a starting point for PCA and differential gene expression analysis. As anticipated, PCA showed a clear separation between muscle and adipose genes in controls (Figure 2a) and PDAC (Figure 2b). However, there was no clear separation between controls and PDAC when muscle (Figure 2c) and adipose (Figure 2d) tissues were plotted separately. Differential gene expression analysis between controls and cancer patients identified $961 \mathrm{DE}$ genes in muscle and $2000 \mathrm{DE}$ genes in adipose at a fold change of 1.4 and $p$-value of 0.05 . The volcano plot shows the DE genes in muscle (Figure 3a) and adipose (Figure 3b). Overall, 190 genes were common between muscle and adipose which represents a $~ 7 \%$ overlap between the two tissues (Figure $3 \mathrm{c}$ ), highlighting the tissue specific gene expression patterns. The complete list of DE genes for muscle and adipose is given in Table S1 and Table S2, respectively.

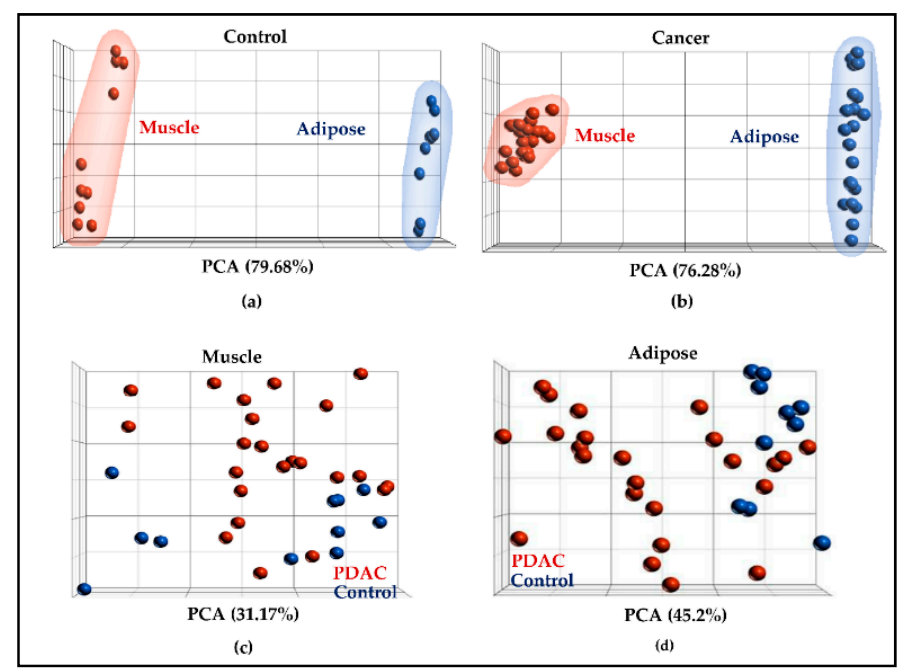

Figure 2. Principal component analysis. All the profiled genes were utilized for the analysis. $(\mathbf{a}, \mathbf{b})$ Indicate that gene expression gene signatures between muscle (a) and adipose (b) were indeed different in controls and PDAC; (c,d) illustrate the PCA within muscle (c) and adipose in controls and PDAC (d). 


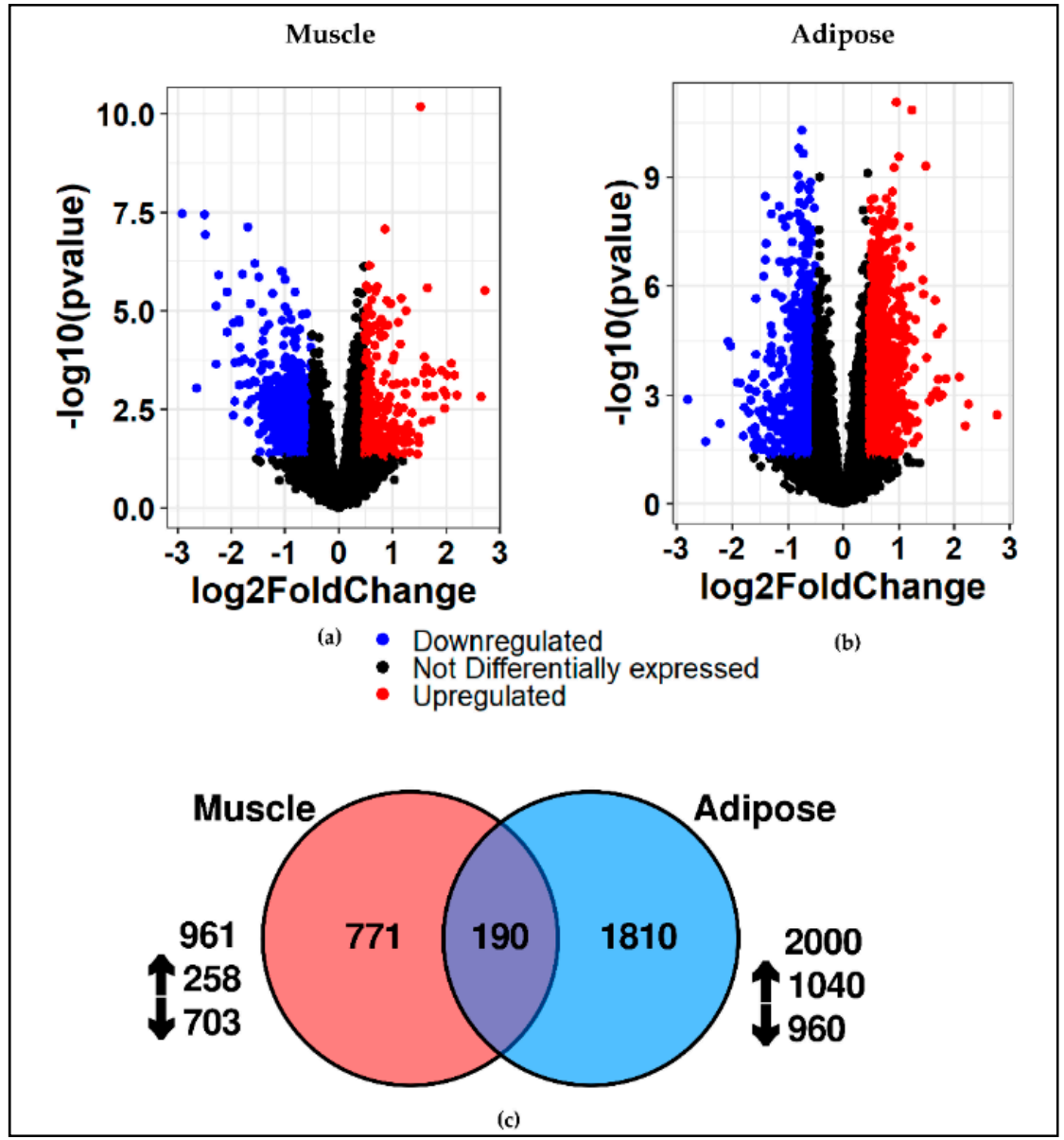

Figure 3. Identification of differentially expressed genes in muscle and adipose. Volcano plot showing the differentially expressed genes for muscle $(\mathbf{a}, \mathbf{b})$ adipose at 1.4 fold change and $p$-value of 0.05 . Red indicates upregulation and blue indicates downregulation; (c) the common differentially expressed genes between muscle and adipose is $\sim 7 \%$.

\subsection{Different Genes Are Involved in Activating or Inhibiting the Common Pathways in Muscle} and Adipose

Respectively, 47 and 53 pathways were identified for muscle and adipose $(p<0.05$ and Z-score $>1.5$ ). Eight pathways were common between muscle and fat: acute phase signaling, senescence pathway, cardiac hypertrophy, IL-8 signaling, CXCR4 signaling, HMGB1 signaling, GP6 signaling and PDGF signaling (Figure 4a). However, the genes involved in activating or inhibiting these common pathways were predominantly different in muscle and adipose tissue (Figure $4 \mathrm{~b}$ ). Other unique pathways identified in muscle include the STAT3 pathway, HIF1 $\alpha$ Signaling and LXR/RXR activation (Figure 4a). A few representative pathways that appeared only in adipose tissue include IL-6 signaling, mTOR signaling, Leptin signaling, Oncostatin M signaling and JAK/STAT signaling (Figure 4a). The complete list of pathways, along with the genes involved and $p$-values for muscle is given in Table S3 and for adipose in Table S4. 


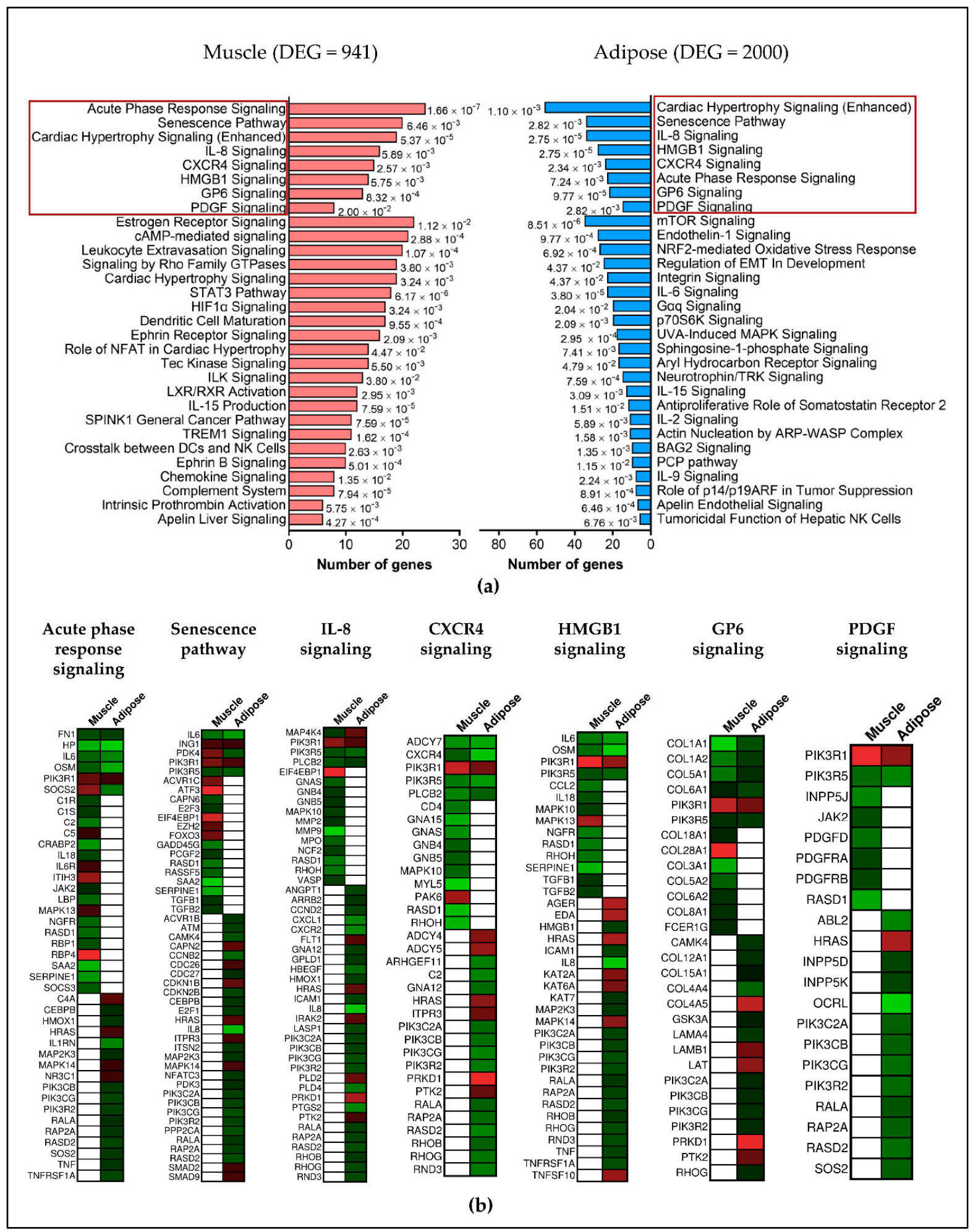

Figure 4. Canonical pathways for muscle and adipose. (a) The top pathways highlighted in red indicates the common pathways between muscle and adipose. The unique pathways are also represented in muscle and adipose; (b) although there are common pathways between muscle and adipose, the genes involved in activating or inhibiting those pathways are predominantly different, indicating a tissue specific gene expression.

To further characterize the different classes of molecules that are expressed in muscle and adipose, we classified the DE genes of muscle and adipose into transcriptional regulators (Figure $5 a$ ), growth factors (Figure $5 b$ ), cytokines (Figure $5 c$ ) and receptors (Figure $5 d$ ) 
using IPA. Indeed, many of these genes in all these classes were tissue specific and few were common between muscle and adipose tissue. DE gene profiles were also subjected to disease pathway analysis. The highest association for both muscle (Figure 6a) and adipose (Figure 6b) was "organismal death".

From these different levels of analyses, it is clear that (i) tissue specific expression patterns exist at gene and pathway levels between adipose and muscle, (ii) adipose tissue gene expression appears to be more dynamic than skeletal muscle in PDAC as the number of adipose genes is approximately twice more than muscle, (iii) inflammation is one of the key drivers of both muscle and adipose wasting, and (iv) the cachexia signature is well-established in muscle and adipose even in patients with early stage, resectable pancreatic cancer.

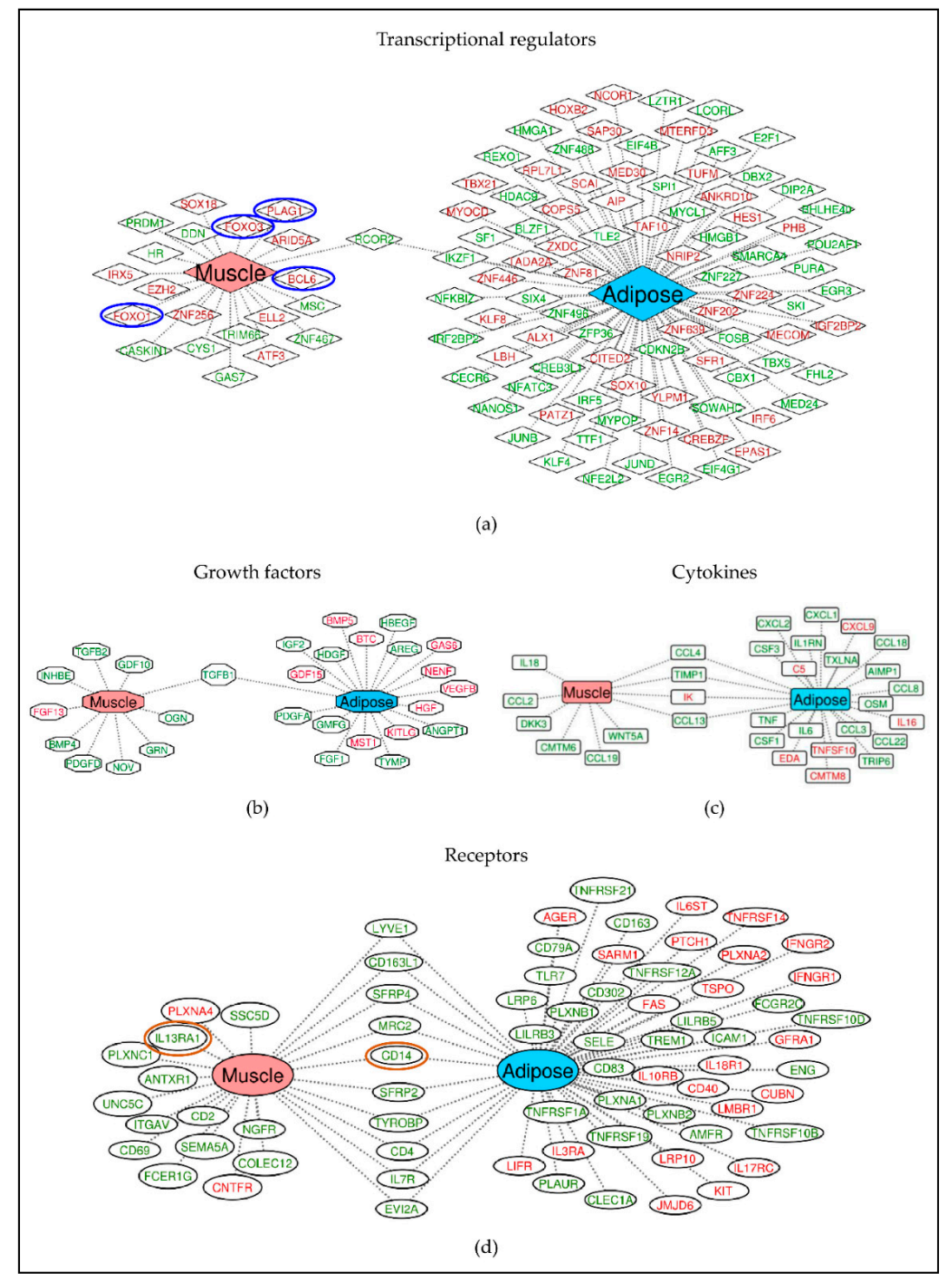

Figure 5. Tissue specific expression of different classes of molecules including transcriptional regulators (a), growth factors (b), cytokines (c) and receptors (d). Differentially expressed genes were given as input in IPA and the genes were classified based on their known functions. A strong tissue specific gene expression pattern exists across classes. The genes in red text are upregulated and green text are downregulated genes. The genes represented in between are commonly expressed between muscle and adipose. The genes that are circled in red and blue are also present in aged muscle dataset where the blue circles indicate same direction of effect (upregulated) and red indicates opposite direction of effect (up in one and down in other). 


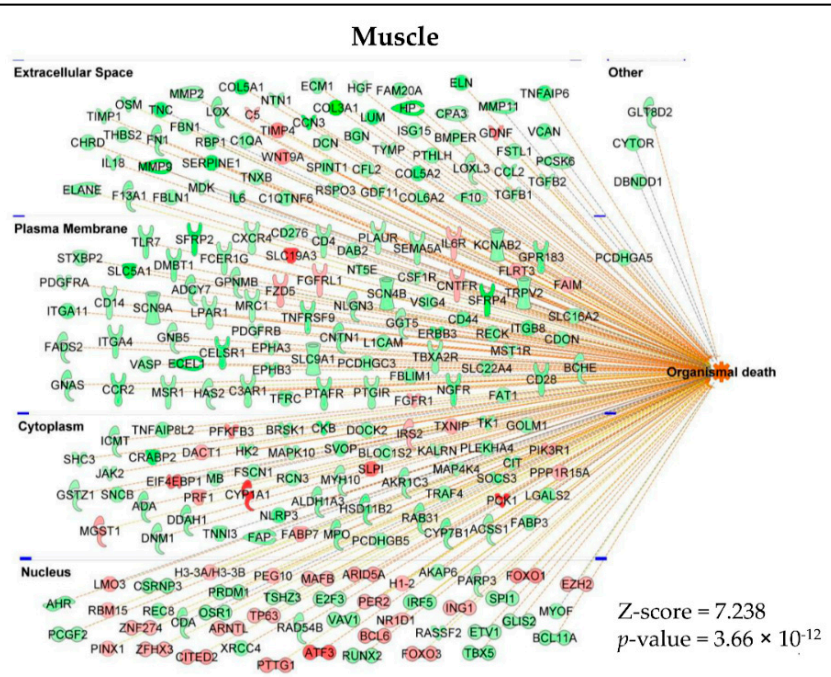

(a)

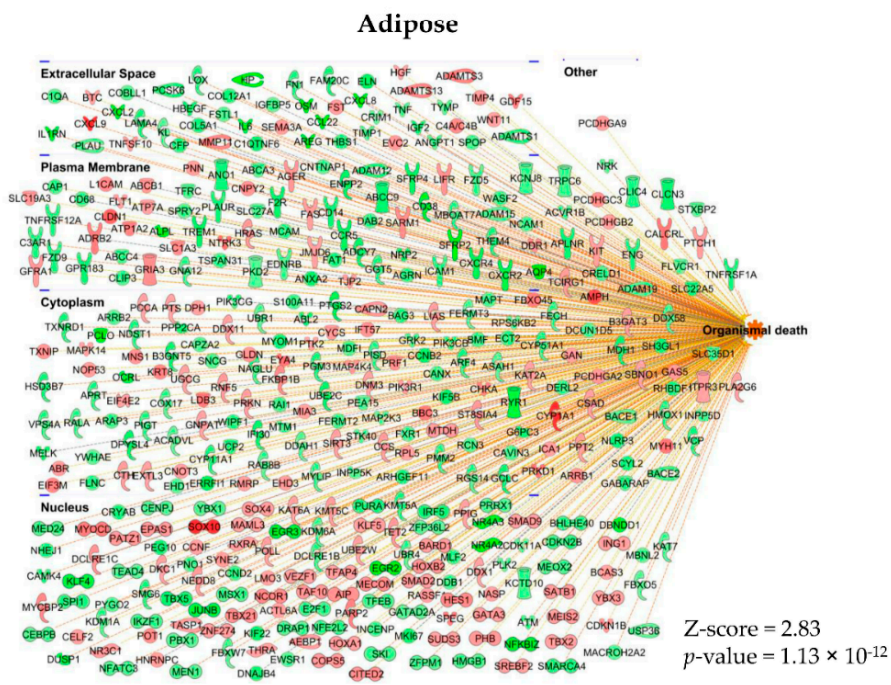

(b)

Figure 6. Gene expression predicted organismal death. Genes predicted to enhance (orange lines) or inhibit (blue lines) organismal death versus no prediction (yellow lines) in muscle (a) and adipose (b).

\subsection{Correlation of Genes from Muscle and Adipose to Cancer Weight Loss Grade}

It is known that combination of weight loss and BMI can differentiate the severity of cachexia and survival [2]. Therefore, to understand whether the genes relate to degree of weight loss, we used all the profiled genes from muscle and adipose and correlated them to cancer weight loss grade, a score generated from BMI and 6-month history of weight loss that has prognostic value in patients with advanced cancers. Only PDAC samples were used for this analysis. To further confirm if these genes are correlated to PDAC alone, we also ran the correlation for controls and removed the genes that were common between PDAC and controls from further interpretation. Genes that correlated with $\mathrm{r}>0.5$ and $p<0.05$ were then subjected to clustering analysis ( $\mathrm{k}$-means clustering) using the STRING database.

A total of 340 genes from muscle, including several known genes for cachexia such as FOXO1, FOXO3, PIK3RI, GLUL were correlated with CWLG (Figure 7a and Table S5). Other genes which were not previously implicated such as PPP1R8, WNT9A, SESN1, CCDC68, RNF207, POMT2 and DST were also found to be correlated with CWLG. KDM6B and FOXO1 were identified as top nodal molecules in the network (Figure $7 \mathrm{~b}$ ). 


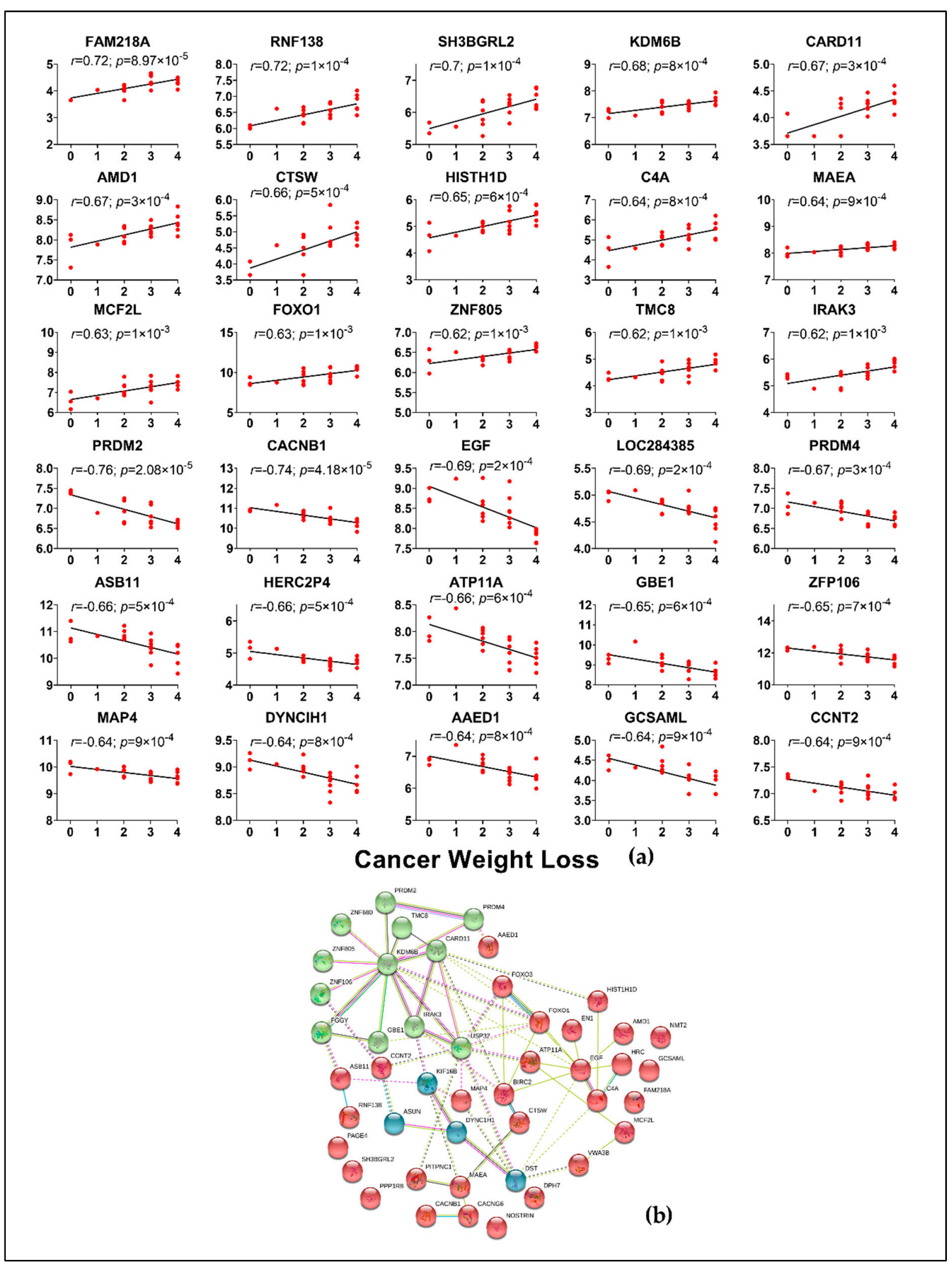

Figure 7. Top 30 muscle genes correlated with CWLG and gene network. (a) All genes were correlated against CWLG with PDAC samples alone. Spearman's rank correlation was performed and only genes with $\mathrm{r}>0.5$ and $p<0.05$ were considered. 340 genes correlated with CWLG; (b) the network was generated using the STRING database for the top 50 correlated genes with CWLG. Orphan networks were removed.

A total of 98 DE genes from adipose tissue were correlated with CWLG and not surprisingly given the relative paucity of data in adipose tissue, the roles of these genes in the context of PDAC and cachexia are undescribed. The top representative genes include 
RPS4X, AFF3, PDZD8 and DBX2 (Figure 8a and Table S6). APOE was identified as the top molecule in the network (Figure $8 \mathrm{~b}$ ).

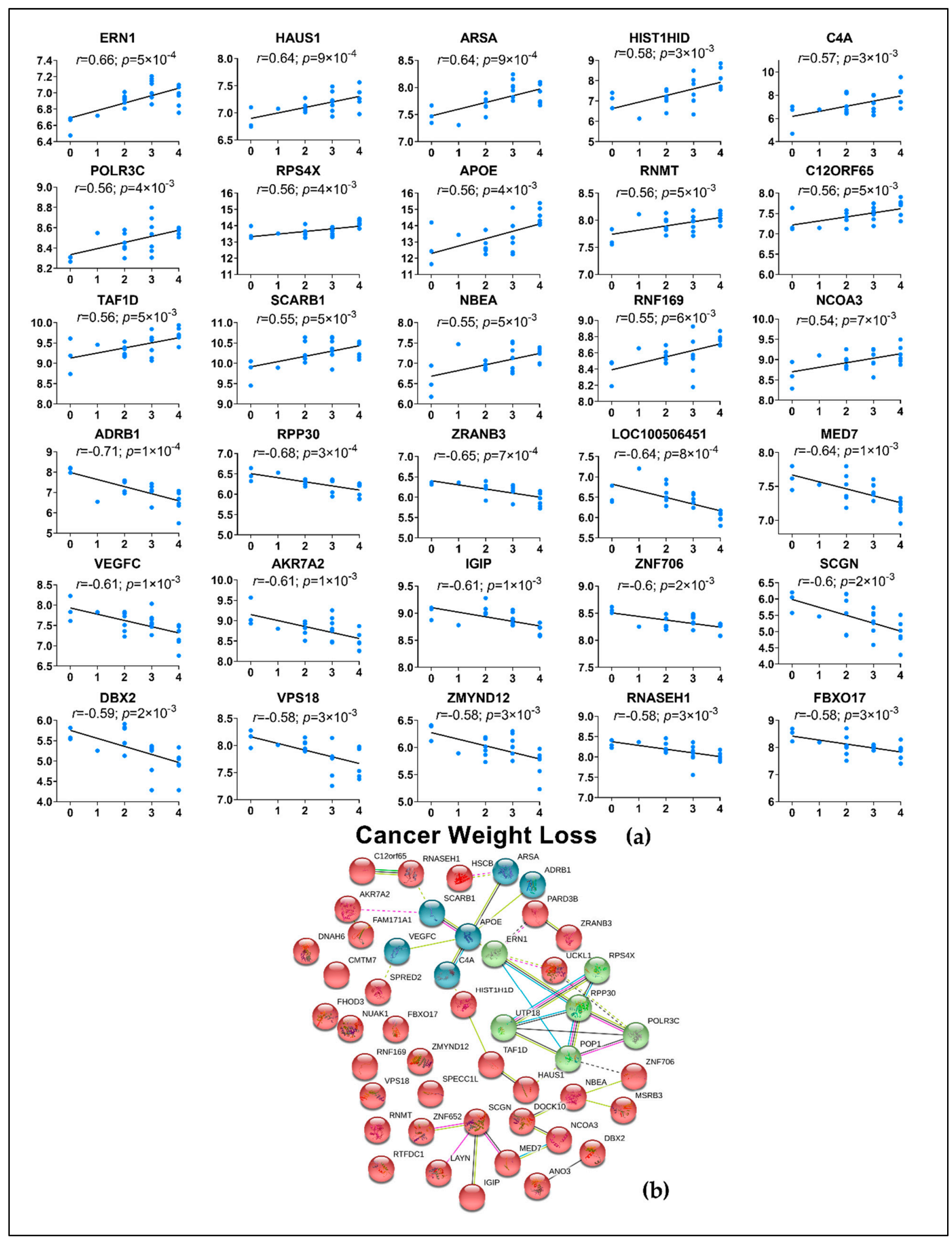

Figure 8. Top 30 adipose genes correlated with CWLG and gene network. (a) All genes were correlated against CWLG with PDAC samples alone. Spearman's rank correlation was performed and only genes with $\mathrm{r}>0.5$ and $p<0.05$ were considered and 98 genes correlated with CWLG; (b) the network was generated using the STRING database for the top 50 correlated genes with CWLG. 


\subsection{Comparison with External Datasets}

We sought to validate our data with external datasets. Few human studies are available with moderate sample size in muscle and only one study has profiled adipose wasting in cachexia to date. Since many of the human studies in cachexia have used array platforms, technological advancement must be considered in deciding the cut-off for the datasets. For example, ion proton sequencing has higher sensitivity and orders of magnitude of detection when compared to microarray [26]. One aim of this analysis was to identify common gene signatures or drivers associated with cachexia across different cancer types. In muscle, 2481 genes were identified in our IU dataset (Table S7), while 1737 genes were identified in the external dataset GSE18832 (Table S8). 294 genes were common between both the datasets, of which 251 genes had similar direction of effect in both datasets ( $~ 84 \%)$ (Figure 9). In adipose, the IU dataset had 4744 genes (Table S9) while the external dataset GSE20571 had 1372 genes (Table S10). 426 genes were common between both the datasets, of which $357(\sim 83 \%)$ genes showed similar direction of effect in both the datasets (Figure 9).

Interestingly, many functionally validated genes in muscle wasting from model systems and humans such as FOXO1, FOXO3 [27], PDK4 [28], IL6R [29], PIK3R1 [7], LIF [30], and SLC39A14 (also known as ZIP14) [31] were identified in both the human skeletal muscle datasets. The top pathways include regulation of eIF4 and p70S6K signaling, mTOR signaling, and insulin receptor signaling. In adipose, inflammation and its related pathways were highly enriched. Pathways such as acute phase response signaling, IL-8 signaling, IL-10 signaling, complement system, Toll-like receptor signaling, GP6 signaling were identified. The complete lists of common genes and pathways for the muscle and adipose datasets are found in Tables S11 and S12, respectively.

It is well documented that gene expression significantly changes with age. As age was significantly different between our control and PDAC, we wanted to understand the extent of gene expression changes common between aging and PDAC. To address this, we identified a non-cancer muscle microarray dataset (GSE9676, GPL 96) which had young (20-30 years old, $n=14$ ) and old participants (65-75 years old, $n=16$ ) in the study. Only baseline samples were considered for analysis. We considered DE genes with 1.2-fold change and $p<0.1$ and identified 1968 genes. When overlaid with IU muscle dataset, only 294 genes were common between IU dataset and GSE9676 age dataset (Table S13), while 1674 genes were unique to age and 2229 genes were unique to PDAC muscle (Figure 10). Interestingly, many of the cachexia associated genes such as FOXO1, FOXO3, SIRT1, SMAD3 and FABP3 were identified in age related gene expression in similar direction. Similarly, at the pathway level, only six pathways were common between the two datasets. There was minimal overlap of genes between cachexia and age (Figure 11) suggesting that mechanisms of age-related muscle loss could be strikingly different from cancer related muscle wasting. We did not find any age-related human gene expression dataset for adipose tissue and therefore we restricted the analysis to muscle. The list of significant pathways for PDAC muscle and GSE9676 is given in Table S14 and Table S15, respectively. 


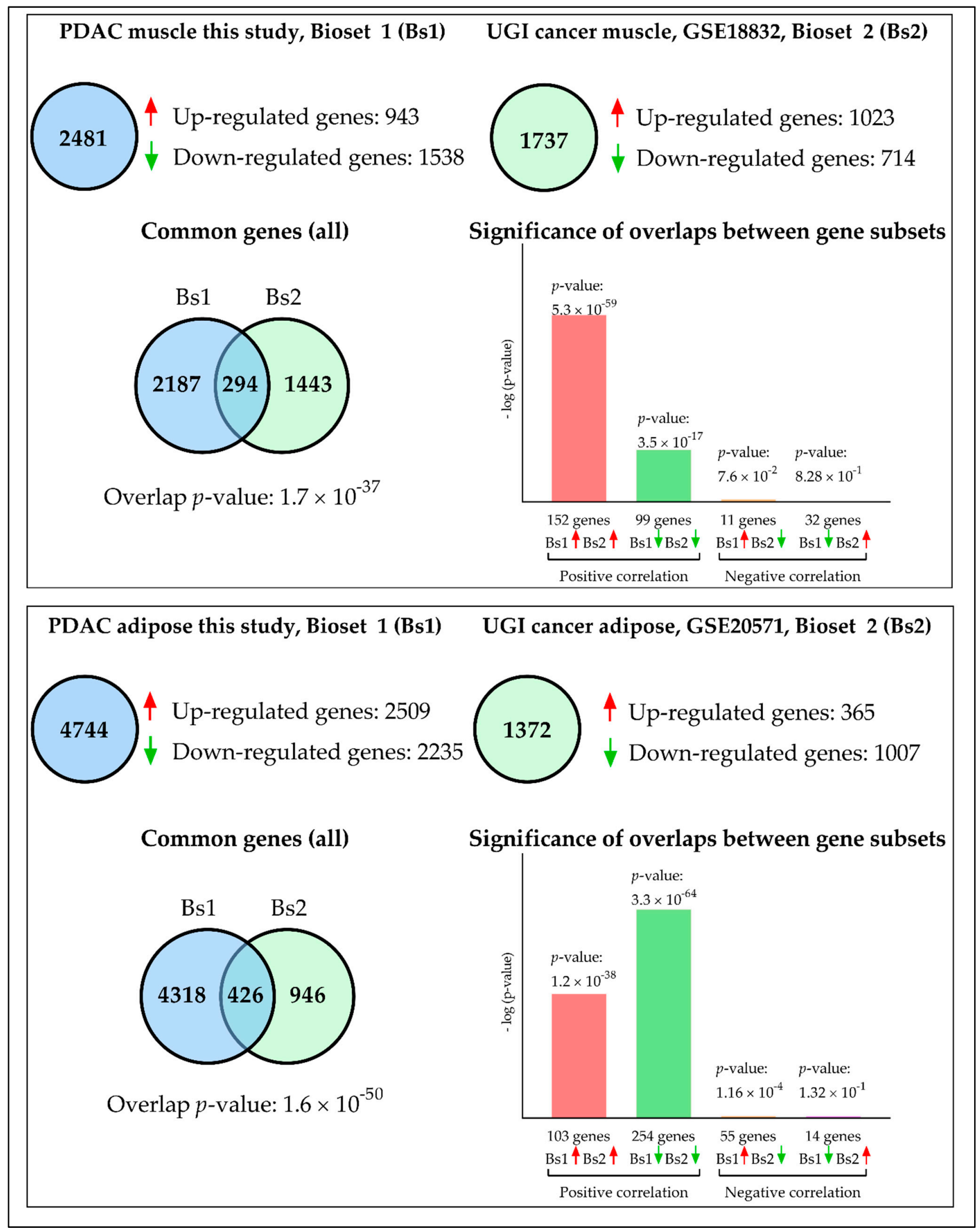

Figure 9. Validation of muscle and adipose DE genes in external dataset. For muscle, 294 genes were common between IU and the external dataset, of which 251 genes had similar direction of effect ( $84 \%$ ). For adipose tissue, 426 genes were common between the two datasets of which 357 genes had similar direction of effect $(83 \%)$. 


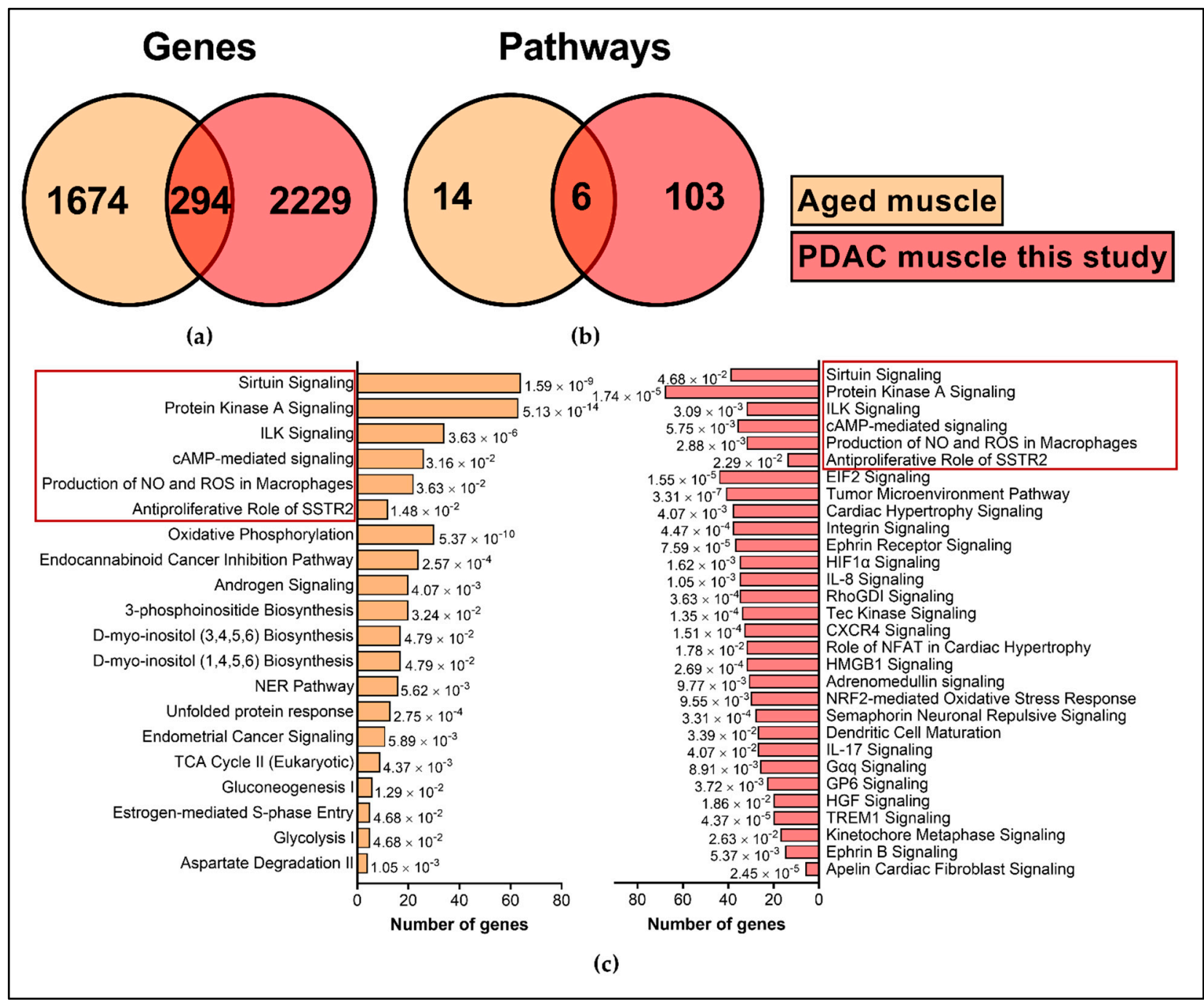

Figure 10. Comparison of age-related transcriptome against PDAC muscle. (a) For muscle, 294 genes were common between PDAC muscle (this study) and GSE9676. (b) There was a minimal overlap between pathways. (c) The list of significant pathways with z-score of 1.5 and $p<0.05$ are represented for GSE9676 and top 30 pathways in PDAC muscle. The common pathways between the two datasets are highlights in red. 


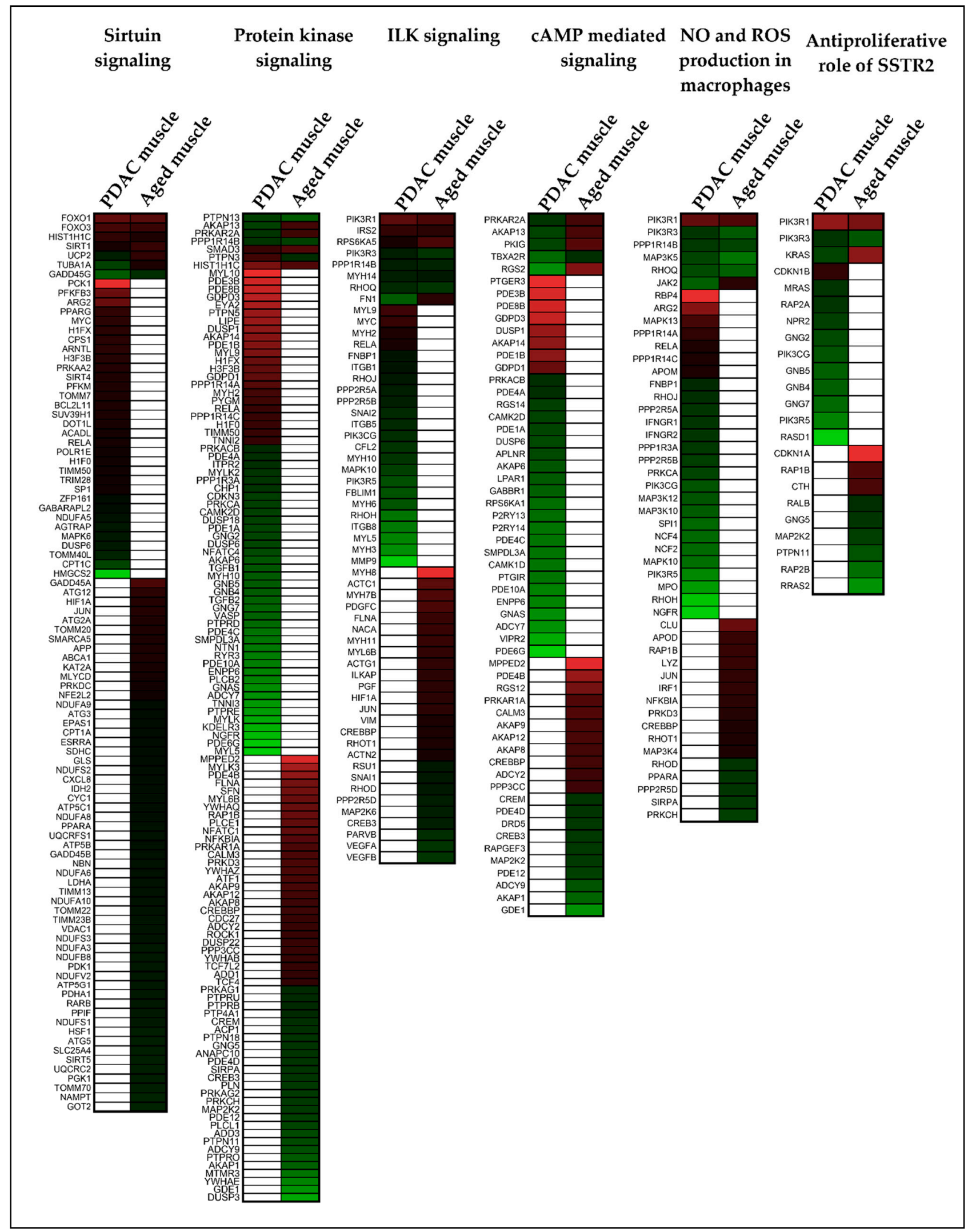

Figure 11. Comparison of age-related transcriptome against PDAC muscle. Six Common pathways were identified between GSE9676 and IU PDAC muscle dataset. Heatmap for each pathway indicate that genes identified in aged muscle dataset and PDAC muscle wasting dataset are predominantly different. Red and green color represent up and downregulated genes, respectively. 


\section{Discussion}

This is the first study to report distinct muscle and adipose gene expression profiled from the same patients having same cancer type, PDAC. Although several common pathways were identified between muscle and adipose, the genes involved in these pathways are predominantly different, suggesting that adipose and muscle wasting may be mediated by independent mechanisms. When examining only the patients with cancer, few well known genes and many unknown genes in muscle were found to be highly correlated with degree of cancer weight loss grade. A similar inference cannot be made from the adipose correlation as this is the first study to profile adipose tissue from a homogeneous cohort and the second overall in the literature to profile human genes associated with fat wasting in cachexia. Although the mechanism through which adipose loss occurs in cachexia has been studied in preclinical models [32-36], the specific mechanisms involved in adipose changes in human PDAC cachexia has not been studied till date. We also directed our efforts in validating the signatures in different cancer type from a different geographic location. The fact that some of the well-studied genes in animal models of cachexia are validated in these human datasets is highly promising. Of course, many genes identified in the muscle and adipose validation data point to inflammation, which is a well-established component of cachexia.

As much as the distinct gene expression profiles between muscle and adipose seem obvious, it conveys a critical information that adipose tissue may have independent mechanisms in causing fat wasting providing a potential therapeutic opportunity to target adipose wasting. This was evident from our pathway results where similar pathways had predominantly different and few common genes between adipose and muscle. One possibility for the overall difference in gene expression in adipose versus muscle is that adipose wasting might be well underway prior to overt muscle loss in PDAC cachexia. Although the majority of cachexia research has focused on muscle, our recent clinical study showed that fat loss alone is associated with reduced survival [4]. Furthermore, mechanistic studies in animal models suggest that blocking lipolysis can promote muscle preservation in cachexia $[34,37,38]$. The molecular mechanisms behind adipose wasting in cancer remain unclear. Our pathway analysis and comparison to the diabesity literature indicate that inflammation could be one of the key drivers of adipose wasting. Indeed, interleukin signaling pathways such as IL-2, IL-6, IL-8, IL-9, IL-15 and IL-23 were observed in adipose. While IL-6 is known to cause fat wasting in cachexia [39], more experimental evidence is needed to understand the interaction between cytokines in augmenting adipose wasting in cachexia. Some of these pro-inflammatory cytokines such as IL-6, IL-8 and IL-23 are implicated in tumor progression and metastasis. IL-4, IL-10 and chemokines recruit circulating monocytes leading to generation of tumor associated macrophages (TAM). The infiltrated monocytes when primed in tumor microenvironment release pro-inflammatory cytokines thereby causing a cachectic environment. However, the exact mechanism through which the macrophages modulate adipose tissue mechanisms in cachexia remains to be elucidated [40]. In another interesting observation, mTOR and NF-kB signaling, which have long been implicated in muscle growth and wasting [41-44], were identified in adipose tissue here and are known to regulate lipogenesis and lipolysis respectively [45] [46,47]. Based on the IPA, mTOR signaling has a z-score of -2.4, indicating pathway inhibition. The implication of this is unclear, given that the role of mTOR in adipose tissue in cachexia has thus far been little explored.

There is more than one-way that adipose tissue can have a negative impact on skeletal muscle and survival. Evidence suggests that lipids may have an important role in maintaining skeletal muscle mass and on the flip side, it can also affect muscle function. Pathologic accumulation of fat in muscle can cause myosteatosis but the exact mechanisms is not clear, although few genes have been studied in injury model. Increased expression of FABP4, a fatty acid carrier protein leads to accumulation of fat in a muscle injury model [48], and FABP4 is indeed upregulated in our IU PDAC muscle and external datasets. Myosteatosis alone is associated with increased mortality in cancer [49] and Stretch et al., showed 
that myosteatosis, sarcopenia and the combination are associated with reduced survival in pancreatic and periampullary adenocarcinomas [50]. This suggests that myosteatosis in cachexia affects survival and their molecular mechanisms in cancer cachexia should be delineated. The key conclusions from these findings are: (i) it is critical to study the molecular mechanisms of adipose wasting along with muscle because fat only loss phenotype is possible at least in PDAC cachexia at this point, (ii) mitigating fat loss may also reduce the incidence of myosteatosis thereby improving the overall survival of patients and (iii) many pathways that are extensively studied in muscle wasting but never in adipose wasting were identified and should be investigated further to see if it can lead to newer therapeutic interventions.

For any study, validating the findings in an external dataset adds better value to the existing findings. However, with only a limited number of human studies conducted in cachexia to date, finding well-matched datasets would be ideal but never a reality. The external datasets were profiled using rectus abdominis muscle and subcutaneous adipose tissue similar to our study but were collected from multiple cancer types and using different gene profiling platforms. While the GSE18832 muscle study compared non-cancer controls to cachectic cases as we did, the GSE20571 adipose study compared weight-stable cancer controls to cachectic cases. Given these differences, the common genes may more likely represent true observations of cachexia-specific events. It is most promising that genes such as IL-6R, FOXO1, PDK4, and ZIP14, among others, that have been functionally validated in animal models were identified in both ours and external datasets. Blocking the overexpression of these molecules were shown to reverse muscle wasting and had beneficial effects. Therapeutics targeting FOXO signaling pathways and IL6-R are in various stages of testing in pancreas cancer [51,52], but whether these might have beneficial impact on cachexia needs to be investigated. Given there are many disparities between animal models and human findings in cachexia, findings from human studies must be tested in animal models towards building for a possible trial. To have genes with therapeutic potential expressed in common between humans and model systems augurs well for the future of cachexia research. More efforts are required to understand the mechanisms involved in adipose wasting in cachexia.

In our study cohort, age was significantly different between cases and controls. It is well documented that gene expression changes with age [53-55]. To address the influence of age-related gene expression in our study, we analyzed age related muscle gene expression dataset and compared it to our IU PDAC muscle data. Our results showed fewer overlapping genes and more unique genes, suggesting that biological aging process could have different mechanism compared to cancer associated muscle wasting. A similar trend was observed in pathways as well. The key differences in the molecular mechanisms can be attributed to the presence of cancer and its associated chemotherapy effects. It is known that cancer and its related treatment can lead to an accelerated aging process [56]. As well, the effect of chemotherapy is also attributed to accelerated aging as telomere shortening was exacerbated in older patients subjected to chemotherapy $[57,58]$. One of the common pathways identified was the sirtuin signaling pathway, which has few of the well characterized cachexia genes such as FOXO1 to be present in aging. FOXO family of transcription factors plays an important role in aging and longevity. One of the key roles of FOXO factors is to modulate the ubiquitin proteasome pathway which has been studied in cancer cachexia. In cachexia, activation of FOXO leads to increased activation of ubiquitin ligases leading to proteolysis and eventually muscle wasting [59,60]. FOXO1 was also upregulated in age related gene expression. It could be surmised that FOXO1 could act through the same ubiquitin mechanisms in causing muscle loss in aging which requires further investigation. With loss of muscle mass being central to both aging and cachexia, and with evidence suggesting that inhibition of FOXO transcriptional activity attenuates muscle wasting [27], it could be an interesting target to pursue for therapeutics.

The study has limitations. The differential gene expression between controls and cancer subjects is confounded by the age difference, which we have attempted to address 
by using an external transcriptome dataset. We will aim to avoid this in our future studies. Another way to address this issue is to perform gene expression between weight stable versus weight losing cancer patients. However, we may not know if the weight stable cancer patients continued to remain weight stable, unless we have a longitudinal follow up of patients, which we lack in this study. As well, in our current study, we have only three weight loss grade 0 patients with PDAC and performing differential expression with such small numbers can lead to compromised results. However, the correlation analysis for cancer weight loss grade were done using cancer patients only and are thus not confounded. Furthermore, we did not analyze the data for sex specific gene differences due to the small sample size. As well, there are different phenotypes observed in cachexia such as muscle only loss, fat only loss or a combination of both, which could not be addressed in this analysis due to the lack of longitudinal body composition data.

\section{Materials and Methods}

\subsection{Recruitment of Study Subjects}

The study was conducted under the Indiana University Institutional Review Board (IRB) approved protocol \#1312105608, the same protocol referenced in our prior study [61]. Patients were recruited at Indiana University Hospital between 2014 and 2016. Written and informed consent was obtained. The cancer cohort consists of 24 patients with PDAC-23 had localized PDAC and underwent surgery and 1 participant had chemotherapy prior to surgery. Patients within the control group underwent surgery for non-malignant conditions including hernia repair and cholecystectomy. Rectus abdominis muscle from the incision site and adjacent subcutaneous adipose tissues were collected and snap frozen in liquid nitrogen and stored at $-80^{\circ} \mathrm{C}$ until further use. In all, we had muscle and adipose tissues from 24 PDAC subjects and 11 non-cancer controls.

\subsection{Body Composition Measurements Using Computed Tomography}

Body composition measurements were obtained from the CT scan obtained as a part of standard of care. The third lumbar vertebrae were used as a landmark to measure the skeletal muscle area $\left(\mathrm{cm}^{2}\right)$, total adipose area $\left(\mathrm{cm}^{2}\right)$ and skeletal muscle density $[62,63]$. Skeletal muscle index and total adipose index were calculated by normalizing surface area to height $\left(\mathrm{cm}^{2} / \mathrm{m}^{2}\right)$. Image analysis was performed using the SliceOMatic software. History of weight loss was obtained from patients using the patient-generated subjective global assessment (PG-SGA) $[64,65]$ and confirmed by consulting the medical record. Cancer weight loss grade (CWLG) was calculated using 6-month weight loss history and BMI as described by Martin et al. [2].

\subsection{Isolation of $R N A$}

RNA was isolated using AllPrep DNA-RNA-miRNA Universal Kit (80224, Qiagen, Valencia, CA, USA). An approximately $10 \mathrm{mg}$ section was removed from the main biopsy in a minimal amount of time. All surfaces were RNase-free. The biopsies were returned to dry ice while the excised piece was homogenized using the TissueRuptor Homogenizer for $30 \mathrm{~s}$ in RLT plus buffer. Homogenized lysates were frozen at $-80^{\circ} \mathrm{C}$ until ready for complete isolation. For muscle, RNA isolation was performed on the Qiacube Instrument using the "Purification of DNA from Tissues or Cells; Part A" and "Purification of RNA, including miRNA, from tissues or cells; Part B" Protocol Sheet. Default elution values were used. RNA was quantified using the RNA-BR Kit and Qubit Fluorometer. For adipose, RNA isolation was performed manually due to the chloroform step in the isolation protocol. Samples were quantified and sized using the Qubit BR RNA Kit and TapeStation RNA Kit.

\subsection{Library Preparation}

For muscle, library preparation was done in accordance with AmpliSeq Transcriptome Human Gene Expression Kit (A26325, Thermo-Fisher Scientific, Waltham, MA, USA, MAN00010742 Rev. A.0) using 10 ng of RNA as starting material. Libraries for all samples 
were prepared simultaneously and barcoded using Ion Xpress Barcode 1-16 Kit (4471250, Thermo-Fisher Scientific). Libraries were quantified using the Ion TaqMan Quantitation Kit (4468802, Thermo-Fisher Scientific) in Fast Mode using the 7900HT (Thermo-Fisher Scientific). Libraries were diluted to $75 \mathrm{pM}$ for sequencing. For adipose, automated library preparation from $10 \mathrm{ng}$ of RNA was completed according to the Ion Chef protocol (MAN0013432). Libraries were barcoded using the plates available with the Chef Kit and diluted to $50 \mathrm{pM}$ for sequencing. For both tissues, template preparation and sequencing were performed using the Ion PI Hi-Q Chef Kit (A27198, Thermo-Fisher Scientific) with template quantitation using the IonSphere Quality Control Kit (4468656, Thermo-Fisher Scientific).

\subsection{Differential Gene Expression}

Raw reads were obtained by mapping the sequence to the human genome build, hg19. Principal component analysis (PCA) was performed using Partek flow genomic analysis software. DESeq2 was used to identify differentially expressed (DE) genes between control and PDAC using $\mathrm{R}$ for both adipose and muscle gene expression analyses [66]. For discovery purposes, a fold change of 1.4 and $p$-value of 0.05 were used to identify DE genes. For PCA and for performing correlation with CWLG, DESeq2-generated variance stabilization counts were used. We refer our dataset as IU datasets which was generated for the study from Indiana University.

\subsection{Statistical Analysis}

Student's $t$-test was used to compare age, BMI, skeletal muscle index and total adipose index between subject groups. Chi-square test was used for gender comparison and Fisher's exact test for cancer associated weight loss between non-cancer controls and PDAC cases. Pearson correlation test was used for correlating all the muscle and adipose genes with CWLG. As we wanted to consider only the strong correlations, genes with Pearson $r \geq 0.5$ and $p<0.05$ were selected for downstream analyses. Correlation plots were generated using GraphPad Prism 7. The raw reads and normalized counts for all the samples are deposited in Gene Expression Omnibus database (GSE133979). The overall workflow is presented in Figure 1.

\subsection{External Validation Datasets}

Two datasets from Gene Expression Omnibus data repository were used as contrasts to our own datasets. GSE18832 profiled the rectus abdominis transcriptome of 3 non-cancer controls and 18 upper gastrointestinal cancer subjects via Affymetrix HGU133plus2 GeneChip array [67]. GSE20571 profiled abdominal subcutaneous adipose tissue of 14 weight stable cancer controls and 13 weight losing cancer patients using Affymetrix Gene 1.0 ST Array [21]. Factoring in the cross-platform differences and discovery intent, we used a relaxed cut-off of 1.2-fold change and $p<0.1$, for both our dataset and the external dataset for differential gene expression. Datasets were compared using Illumina BaseSpace Correlation Engine [68]. The gene numbers correspond to the output obtained from the correlation engine software.

As age was significantly different between the cases and controls in our study, we wanted to understand if there are any common genes between cachexia and age. We identified a muscle gene expression dataset (GSE9676) profiled using vastus lateralis muscle, that had 14 young and 16 older participants. The "analyze GEO2R" tool in gene expression omnibus database is used for differential expression analysis. We considered only the baseline samples for analysis. There was no human adipose dataset available for age. In line with the other external datasets, we selected differentially expressed genes with 1.2-fold change and $p<0.1$.

\subsection{Pathway Analysis for DE Genes and Gene Networks}

Ingenuity Pathway Analysis (IPA, Qiagen, Version 2.3, November 2018) was used for pathway discovery, identification of upstream regulators, and classification of genes 
with fold change $>1.4$ and $p$-value of 0.05 for both muscle and adipose. Pathways with Z-score $\geq 1.5$ and $p<0.05$ were considered significant. We focused mainly on signaling pathways for muscle and adipose. However, the entire list of pathways is presented in the supplementary information. STRING database version 11.0 was used to generate gene networks for genes that were significantly correlated with CWLG.

\section{Conclusions}

This is the single largest study in cachexia to generate expression profiles of muscle and adipose genes from the same individuals and from the same cancer type to understand the concurrent muscle and adipose wasting in a homogenous population.

Supplementary Materials: The following are available online at https://www.mdpi.com/article/ 10.3390/cancers13081975/s1: Table S1: List of differentially expressed genes in muscle, Table S2: List of Differentially expressed genes in adipose, Table S3: Canonical pathways for muscle using the DE genes, Table S4: Canonical pathways for adipose using the DE genes, Table S5: Complete list of muscle genes correlated with CWLG at $\mathrm{r} \geq 0.5$ and $p<0.05$, Table S6: Complete list of adipose genes correlated with CWLG at $\mathrm{r} \geq 0.5$ and $p<0.05$, Table S7: IU muscle differentially expressed genes at 1.2 Fold change and $p<0.1$, Table S8: Differentially expressed muscle genes in external dataset in GSE 18832 at 1.2 Fold change and $p<0.1$, Table S9: IU adipose differentially expressed genes at 1.2 Fold change and $p<0.1$, Table S10: Differentially expressed adipose genes in external dataset in GSE 20571 at 1.2 Fold change and $p<0.1$, Table S11: Canonical pathways for the common genes between IU muscle and external muscle (GSE18832) datasets, Table S12: Canonical pathways for the common genes between IU adipose and external adipose (GSE20571) datasets, Table S13: Differentially expressed age related genes in external dataset in GSE 9676 common to IU muscle dataset at 1.2-fold change and $p<0.1$, Table S14: Canonical pathways for IU muscle dataset for genes with 1.2 FC and $p<0.1$, Table S15: Canonical pathways for GSE9676 Old vs young dataset for genes with $1.2 \mathrm{FC}$ and $p<0.1$.

Author Contributions: Conceptualization, M.R., M.E.C., L.G.K. and T.A.Z.; methodology, E.P.C., A.N. (Ashok Narasimhan), M.G.H., N.J.Z., C.M.S., K.N.H.S., D.E.I.S., J.K.K., S.S., L.G.K. and T.A.Z.; data curation, X.Z., E.P.A. and B.A.H.; formal analysis, A.N. (Ashok Narasimhan), Y.L., G.J., B.A.H., M.R. and T.A.Z.; resources, A.N. (Atilla Nakeeb) Y.L., L.G.K. and T.A.Z.; writing-original draft preparation, A.N. (Ashok Narasimhan) and T.A.Z.; writing-review and editing, All authors; supervision, M.R., M.E.C., L.G.K. and T.A.Z.; funding acquisition, M.R., M.E.C., L.G.K. and T.A.Z. All authors have read and agreed to the published version of the manuscript.

Funding: This research was funded in part by grants to TAZ from NIH (NCI R01CA194593), the IU Simon Comprehensive Cancer Center Pancreatic Cancer Working Group (NCI P30CA082709), the Veterans Administration (VA I01CX002046), and the Lustgarten Foundation, and by grants to LGK from NIH (NIDDK R01DK096167) and the Lilly Endowment, Inc. We thank the IUSCCC at Indiana University School of Medicine support from the Clinical Trials Office, the Tissue Procurement \& Distribution Core, and the Collaborative Core for Cancer Bioinformatics, resources supported by the IU Simon Cancer Center (NCI Grant P30CA082709), the Purdue University Center for Cancer Research (Grant P30CA023168), and the Walther Cancer Foundation.

Institutional Review Board Statement: The study was conducted according to the guidelines of the Declaration of Helsinki and approved by the Indiana University Institutional Review Board (IRB) approved protocol \#1312105608.

Informed Consent Statement: Written and informed consent was obtained from all subjects involved in the study.

Data Availability Statement: The raw files (bam files) and normalized counts for all the samples are deposited in Gene Expression Omnibus database (GSE133979).

Acknowledgments: The authors acknowledge the assistance of Leigh Mott with IRB protocol development and Cynthia Brooks with patient recruitment and tissue and data collection. The authors would like to thank Vickie Baracos for providing constructive feedback on the manuscript.

Conflicts of Interest: The authors declare no conflict of interest. 


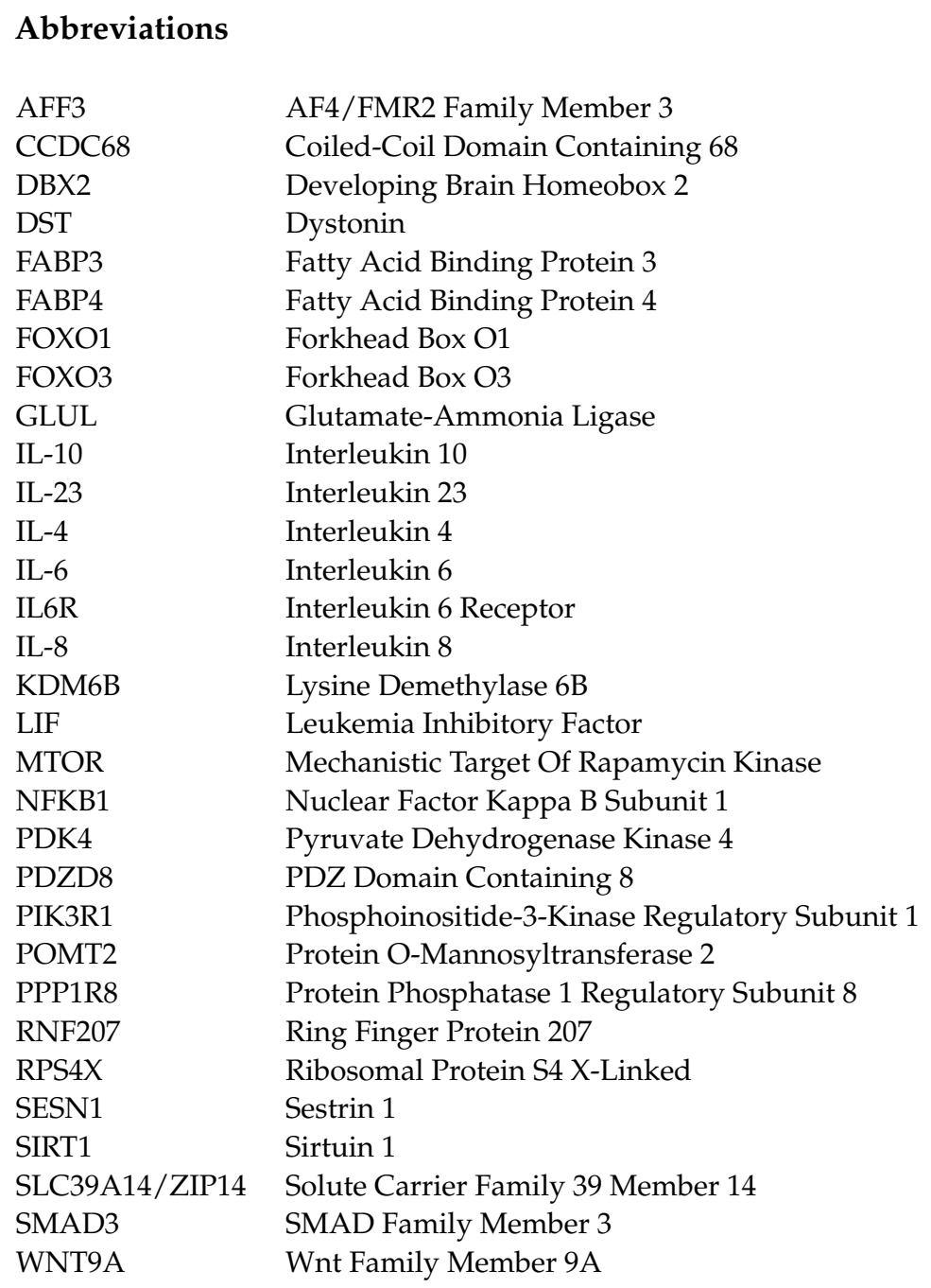

\section{References}

1. Fearon, K.; Strasser, F.; Anker, S.D.; Bosaeus, I.; Bruera, E.; Fainsinger, R.L.; Jatoi, A.; Loprinzi, C.; MacDonald, N.; Mantovani, G.; et al. Definition and classification of cancer cachexia: An international consensus. Lancet Oncol. 2011, 12, 489-495. [CrossRef]

2. Martin, L.; Senesse, P.; Gioulbasanis, I.; Antoun, S.; Bozzetti, F.; Deans, C.; Strasser, F.; Thoresen, L.; Jagoe, R.T.; Chasen, M.; et al. Diagnostic criteria for the classification of cancer-associated weight loss. J. Clin. Oncol. 2015, 33, 90-99. [CrossRef] [PubMed]

3. Ebadi, M.; Mazurak, V.C. Potential Biomarkers of Fat Loss as a Feature of Cancer Cachexia. Mediat. Inflamm. 2015, $2015,820934$. [CrossRef] [PubMed]

4. Kays, J.K.; Shahda, S.; Stanley, M.; Bell, T.M.; O’Neill, B.H.; Kohli, M.D.; Couch, M.E.; Koniaris, L.G.; Zimmers, T.A. Three cachexia phenotypes and the impact of fat-only loss on survival in FOLFIRINOX therapy for pancreatic cancer. J. Cachexia Sarcopenia Muscle 2018, 9, 673-684. [CrossRef] [PubMed]

5. Hendifar, A.E.; Petzel, M.Q.B.; Zimmers, T.A.; Denlinger, C.S.; Matrisian, L.M.; Picozzi, V.J.; Rahib, L.; Precision Promise, C. Pancreas Cancer-Associated Weight Loss. Oncologist 2019, 24, 691-701. [CrossRef]

6. Poulia, K.A.; Sarantis, P.; Antoniadou, D.; Koustas, E.; Papadimitropoulou, A.; Papavassiliou, A.G.; Karamouzis, M.V. Pancreatic Cancer and Cachexia-Metabolic Mechanisms and Novel Insights. Nutrients 2020, 12, 1543. [CrossRef]

7. Mitchell, T.; Clarke, L.; Goldberg, A.; Bishop, K.S. Pancreatic Cancer Cachexia: The Role of Nutritional Interventions. Healthcare 2019, 7, 89. [CrossRef] [PubMed]

8. Flint, T.R.; Fearon, D.T.; Janowitz, T. Connecting the Metabolic and Immune Responses to Cancer. Trends Mol. Med. 2017, 23, 451-464. [CrossRef] [PubMed]

9. Pezzilli, R.; Caccialanza, R.; Capurso, G.; Brunetti, O.; Milella, M.; Falconi, M. Pancreatic Enzyme Replacement Therapy in Pancreatic Cancer. Cancers 2020, 12, 275. [CrossRef]

10. Shyh-Chang, N. Metabolic Changes During Cancer Cachexia Pathogenesis. Adv. Exp. Med. Biol. 2017, 1026, 233-249. [CrossRef]

11. Wang, F.; Liu, H.; Hu, L.; Liu, Y.; Duan, Y.; Cui, R.; Tian, W. The Warburg effect in human pancreatic cancer cells triggers cachexia in athymic mice carrying the cancer cells. BMC Cancer 2018, 18, 360. [CrossRef] 
12. Henderson, S.E.; Makhijani, N.; Mace, T.A. Pancreatic Cancer-Induced Cachexia and Relevant Mouse Models. Pancreas 2018, 47, 937-945. [CrossRef]

13. Zhong, X.; Pons, M.; Poirier, C.; Jiang, Y.; Liu, J.; Sandusky, G.E.; Shahda, S.; Nakeeb, A.; Schmidt, C.M.; House, M.G.; et al. The systemic activin response to pancreatic cancer: Implications for effective cancer cachexia therapy. J. Cachexia Sarcopenia Muscle 2019, 10, 1083-1101. [CrossRef]

14. Bonetto, A.; Aydogdu, T.; Kunzevitzky, N.; Guttridge, D.C.; Khuri, S.; Koniaris, L.G.; Zimmers, T.A. STAT3 activation in skeletal muscle links muscle wasting and the acute phase response in cancer cachexia. PLoS ONE 2011, 6, e22538. [CrossRef] [PubMed]

15. Benny Klimek, M.E.; Aydogdu, T.; Link, M.J.; Pons, M.; Koniaris, L.G.; Zimmers, T.A. Acute inhibition of myostatin-family proteins preserves skeletal muscle in mouse models of cancer cachexia. Biochem. Biophys. Res. Commun. 2010, 391, 1548-1554. [CrossRef] [PubMed]

16. Zimmers, T.A.; Davies, M.V.; Koniaris, L.G.; Haynes, P.; Esquela, A.F.; Tomkinson, K.N.; McPherron, A.C.; Wolfman, N.M.; Lee, S.J. Induction of cachexia in mice by systemically administered myostatin. Science 2002, 296, 1486-1488. [CrossRef]

17. Bonetto, A.; Aydogdu, T.; Jin, X.; Zhang, Z.; Zhan, R.; Puzis, L.; Koniaris, L.G.; Zimmers, T.A. JAK/STAT3 pathway inhibition blocks skeletal muscle wasting downstream of IL-6 and in experimental cancer cachexia. Am. J. Physiol. Endocrinol. Metab. 2012, 303, E410-E421. [CrossRef]

18. Zimmers, T.A.; Fishel, M.L.; Bonetto, A. STAT3 in the systemic inflammation of cancer cachexia. Semin. Cell Dev. Biol. 2016, 54, 28-41. [CrossRef]

19. Talbert, E.E.; Cuitino, M.C.; Ladner, K.J.; Rajasekerea, P.V.; Siebert, M.; Shakya, R.; Leone, G.W.; Ostrowski, M.C.; Paleo, B.; Weisleder, N.; et al. Modeling Human Cancer-induced Cachexia. Cell Rep. 2019, 28, 1612-1622.e4. [CrossRef]

20. Michaelis, K.A.; Zhu, X.; Burfeind, K.G.; Krasnow, S.M.; Levasseur, P.R.; Morgan, T.K.; Marks, D.L. Establishment and characterization of a novel murine model of pancreatic cancer cachexia. J. Cachexia Sarcopenia Muscle 2017, 8, 824-838. [CrossRef] [PubMed]

21. Dahlman, I.; Mejhert, N.; Linder, K.; Agustsson, T.; Mutch, D.M.; Kulyte, A.; Isaksson, B.; Permert, J.; Petrovic, N.; Nedergaard, J.; et al. Adipose tissue pathways involved in weight loss of cancer cachexia. Br. J. Cancer 2010, 102, 1541-1548. [CrossRef]

22. Sun, X.; Feng, X.; Wu, X.; Lu, Y.; Chen, K.; Ye, Y. Fat Wasting Is Damaging: Role of Adipose Tissue in Cancer-Associated Cachexia. Front. Cell Dev. Biol. 2020, 8, 33. [CrossRef]

23. Vaitkus, J.A.; Celi, F.S. The role of adipose tissue in cancer-associated cachexia. Exp. Biol. Med. 2017, 242, 473-481. [CrossRef]

24. Dong, M.; Lin, J.; Lim, W.; Jin, W.; Lee, H.J. Role of brown adipose tissue in metabolic syndrome, aging, and cancer cachexia. Front. Med. 2018, 12, 130-138. [CrossRef] [PubMed]

25. Gallagher, I.J.; Stephens, N.A.; MacDonald, A.J.; Skipworth, R.J.; Husi, H.; Greig, C.A.; Ross, J.A.; Timmons, J.A.; Fearon, K.C. Suppression of skeletal muscle turnover in cancer cachexia: Evidence from the transcriptome in sequential human muscle biopsies. Clin. Cancer Res. 2012, 18, 2817-2827. [CrossRef]

26. Zhang, J.D.; Schindler, T.; Kung, E.; Ebeling, M.; Certa, U. Highly sensitive amplicon-based transcript quantification by semiconductor sequencing. BMC Genom. 2014, 15, 565. [CrossRef] [PubMed]

27. Reed, S.A.; Sandesara, P.B.; Senf, S.M.; Judge, A.R. Inhibition of FoxO transcriptional activity prevents muscle fiber atrophy during cachexia and induces hypertrophy. FASEB J. 2012, 26, 987-1000. [CrossRef]

28. Pin, F.; Novinger, L.J.; Huot, J.R.; Harris, R.A.; Couch, M.E.; O'Connell, T.M.; Bonetto, A. PDK4 drives metabolic alterations and muscle atrophy in cancer cachexia. FASEB J. 2019, 33, 7778-7790. [CrossRef] [PubMed]

29. Narsale, A.A.; Carson, J.A. Role of interleukin-6 in cachexia: Therapeutic implications. Curr. Opin. Support. Palliat. Care 2014, 8, 321-327. [CrossRef] [PubMed]

30. Seto, D.N.; Kandarian, S.C.; Jackman, R.W. A Key Role for Leukemia Inhibitory Factor in C26 Cancer Cachexia. J. Biol. Chem. 2015, 290, 19976-19986. [CrossRef]

31. Wang, G.; Biswas, A.K.; Ma, W.; Kandpal, M.; Coker, C.; Grandgenett, P.M.; Hollingsworth, M.A.; Jain, R.; Tanji, K.; LomicronpezPintado, S.; et al. Metastatic cancers promote cachexia through ZIP14 upregulation in skeletal muscle. Nat. Med. 2018, $24,770-781$. [CrossRef]

32. Agustsson, T.; Ryden, M.; Hoffstedt, J.; van Harmelen, V.; Dicker, A.; Laurencikiene, J.; Isaksson, B.; Permert, J.; Arner, P. Mechanism of increased lipolysis in cancer cachexia. Cancer Res. 2007, 67, 5531-5537. [CrossRef]

33. Arora, G.K.; Gupta, A.; Narayanan, S.; Guo, T.; Iyengar, P.; Infante, R.E. Cachexia-associated adipose loss induced by tumorsecreted leukemia inhibitory factor is counterbalanced by decreased leptin. JCI Insight 2018, 3. [CrossRef]

34. Das, S.K.; Eder, S.; Schauer, S.; Diwoky, C.; Temmel, H.; Guertl, B.; Gorkiewicz, G.; Tamilarasan, K.P.; Kumari, P.; Trauner, M.; et al. Adipose triglyceride lipase contributes to cancer-associated cachexia. Science 2011, 333, 233-238. [CrossRef]

35. Kir, S.; White, J.P.; Kleiner, S.; Kazak, L.; Cohen, P.; Baracos, V.E.; Spiegelman, B.M. Tumour-derived PTH-related protein triggers adipose tissue browning and cancer cachexia. Nature 2014, 513, 100-104. [CrossRef]

36. Petruzzelli, M.; Schweiger, M.; Schreiber, R.; Campos-Olivas, R.; Tsoli, M.; Allen, J.; Swarbrick, M.; Rose-John, S.; Rincon, M.; Robertson, G.; et al. A switch from white to brown fat increases energy expenditure in cancer-associated cachexia. Cell Metab. 2014, 20, 433-447. [CrossRef]

37. Du, L.; Yang, Y.H.; Wang, Y.M.; Xue, C.H.; Kurihara, H.; Takahashi, K. EPA-enriched phospholipids ameliorate cancer-associated cachexia mainly via inhibiting lipolysis. Food Funct. 2015, 6, 3652-3662. [CrossRef] [PubMed] 
38. Argiles, J.M.; Lopez-Soriano, F.J.; Busquets, S. Mechanisms to explain wasting of muscle and fat in cancer cachexia. Curr. Opin. Support. Palliat. Care 2007, 1, 293-298. [CrossRef] [PubMed]

39. Han, J.; Meng, Q.; Shen, L.; Wu, G. Interleukin-6 induces fat loss in cancer cachexia by promoting white adipose tissue lipolysis and browning. Lipids Health Dis. 2018, 17, 14. [CrossRef] [PubMed]

40. Farajzadeh Valilou, S.; Keshavarz-Fathi, M.; Silvestris, N.; Argentiero, A.; Rezaei, N. The role of inflammatory cytokines and tumor associated macrophages (TAMs) in microenvironment of pancreatic cancer. Cytokine Growth Factor Rev. 2018, 39, 46-61. [CrossRef]

41. Yoon, M.S. mTOR as a Key Regulator in Maintaining Skeletal Muscle Mass. Front. Physiol. 2017, 8, 788. [CrossRef]

42. Duval, A.P.; Jeanneret, C.; Santoro, T.; Dormond, O. mTOR and Tumor Cachexia. Int. J. Mol. Sci. 2018, 19, 2225. [CrossRef]

43. Onesti, J.K.; Guttridge, D.C. Inflammation based regulation of cancer cachexia. Biomed. Res. Int. 2014, 2014, 168407. [CrossRef] [PubMed]

44. Gupta, S.C.; Kim, J.H.; Kannappan, R.; Reuter, S.; Dougherty, P.M.; Aggarwal, B.B. Role of nuclear factor kappaB-mediated inflammatory pathways in cancer-related symptoms and their regulation by nutritional agents. Exp. Biol. Med. 2011, 236, 658-671. [CrossRef] [PubMed]

45. Festuccia, W.T. Regulation of Adipocyte and Macrophage Functions by mTORC1 and 2 in Metabolic Diseases. Mol. Nutr. Food Res. 2020, 65, e1900768. [CrossRef]

46. Mao, Z.; Zhang, W. Role of mTOR in Glucose and Lipid Metabolism. Int. J. Mol. Sci. 2018, 19, 2043. [CrossRef] [PubMed]

47. Purohit, J.S.; Hu, P.; Chen, G.; Whelan, J.; Moustaid-Moussa, N.; Zhao, L. Activation of nucleotide oligomerization domain containing protein 1 induces lipolysis through NF-kappaB and the lipolytic PKA activation in 3T3-L1 adipocytes. Biochem. Cell Biol. 2013, 91, 428-434. [CrossRef] [PubMed]

48. Lee, Y.S.; Kim, J.Y.; Oh, K.S.; Chung, S.W. Fatty acid-binding protein 4 regulates fatty infiltration after rotator cuff tear by hypoxia-inducible factor 1 in mice. J. Cachexia Sarcopenia Muscle 2017, 8, 839-850. [CrossRef] [PubMed]

49. Esfandiari, N.; Ghosh, S.; Prado, C.M.; Martin, L.; Mazurak, V.; Baracos, V.E. Age, Obesity, Sarcopenia, and Proximity to Death Explain Reduced Mean Muscle Attenuation in Patients with Advanced Cancer. J. Frailty Aging 2014, 3, 3-8. [CrossRef] [PubMed]

50. Stretch, C.; Aubin, J.M.; Mickiewicz, B.; Leugner, D.; Al-Manasra, T.; Tobola, E.; Salazar, S.; Sutherland, F.R.; Ball, C.G.; Dixon, E.; et al. Sarcopenia and myosteatosis are accompanied by distinct biological profiles in patients with pancreatic and periampullary adenocarcinomas. PLOS ONE 2018, 13, e0196235. [CrossRef]

51. Farhan, M.; Wang, H.; Gaur, U.; Little, P.J.; Xu, J.; Zheng, W. FOXO Signaling Pathways as Therapeutic Targets in Cancer. Int. J. Biol. Sci. 2017, 13, 815-827. [CrossRef]

52. Chen, I.; Johansen, J.S.; Zimmers, T.A.; Dehlendorff, C.; Kirk Parner, V.; Vittrup Jensen, B.; Nielsen, D. PACTO: A single center, randomized, Phase II study of the combination of nab-paclitaxel and gemcitabine with or without tocilizumab, an IL-6R inhibitor, as first-line treatment in patients with locally advanced or metastatic pancreatic cancer. Ann. Oncol. 2017, 28, v209-v268. [CrossRef]

53. Lin, I.H.; Chang, J.L.; Hua, K.; Huang, W.C.; Hsu, M.T.; Chen, Y.F. Skeletal muscle in aged mice reveals extensive transformation of muscle gene expression. BMC Genet. 2018, 19, 55. [CrossRef] [PubMed]

54. Shavlakadze, T.; Morris, M.; Fang, J.; Wang, S.X.; Zhu, J.; Zhou, W.; Tse, H.W.; Mondragon-Gonzalez, R.; Roma, G.; Glass, D.J. Age-Related Gene Expression Signature in Rats Demonstrate Early, Late, and Linear Transcriptional Changes from Multiple Tissues. Cell Rep. 2019, 28, 3263-3273.e3. [CrossRef] [PubMed]

55. Su, J.; Ekman, C.; Oskolkov, N.; Lahti, L.; Strom, K.; Brazma, A.; Groop, L.; Rung, J.; Hansson, O. A novel atlas of gene expression in human skeletal muscle reveals molecular changes associated with aging. Skelet. Muscle 2015, 5, 35. [CrossRef]

56. Hurria, A.; Jones, L.; Muss, H.B. Cancer Treatment as an Accelerated Aging Process: Assessment, Biomarkers, and Interventions. Am. Soc. Clin. Oncol. Educ. Book 2016, 35, e516-e522. [CrossRef]

57. Beeharry, N.; Broccoli, D. Telomere dynamics in response to chemotherapy. Curr. Mol. Med. 2005, 5, 187-196. [CrossRef]

58. Unryn, B.M.; Hao, D.; Gluck, S.; Riabowol, K.T. Acceleration of telomere loss by chemotherapy is greater in older patients with locally advanced head and neck cancer. Clin. Cancer Res. 2006, 12, 6345-6350. [CrossRef]

59. Argiles, J.M.; Busquets, S.; Stemmler, B.; Lopez-Soriano, F.J. Cancer cachexia: Understanding the molecular basis. Nat. Rev. Cancer 2014, 14, 754-762. [CrossRef] [PubMed]

60. Sandri, M.; Sandri, C.; Gilbert, A.; Skurk, C.; Calabria, E.; Picard, A.; Walsh, K.; Schiaffino, S.; Lecker, S.H.; Goldberg, A.L. Foxo transcription factors induce the atrophy-related ubiquitin ligase atrogin-1 and cause skeletal muscle atrophy. Cell 2004, 117, 399-412. [CrossRef]

61. Shahda, S.; Narasimhan, A.; Kays, J.; Perkins, S.M.; Cheng, L.; Hannaford, K.N.; Schloss, D.E.I.; Koniaris, L.G.; Zimmers, T.A. Identification of circulating protein biomarkers for pancreatic cancer cachexia. bioRxiv 2018, 359661. [CrossRef]

62. Martin, L.; Birdsell, L.; Macdonald, N.; Reiman, T.; Clandinin, M.T.; McCargar, L.J.; Murphy, R.; Ghosh, S.; Sawyer, M.B.; Baracos, V.E. Cancer cachexia in the age of obesity: Skeletal muscle depletion is a powerful prognostic factor, independent of body mass index. J. Clin. Oncol. 2013, 31, 1539-1547. [CrossRef] [PubMed]

63. Prado, C.M.; Lieffers, J.R.; McCargar, L.J.; Reiman, T.; Sawyer, M.B.; Martin, L.; Baracos, V.E. Prevalence and clinical implications of sarcopenic obesity in patients with solid tumours of the respiratory and gastrointestinal tracts: A population-based study. Lancet Oncol. 2008, 9, 629-635. [CrossRef] 
64. Bauer, J.; Capra, S.; Ferguson, M. Use of the scored Patient-Generated Subjective Global Assessment (PG-SGA) as a nutrition assessment tool in patients with cancer. Eur. J. Clin. Nutr. 2002, 56, 779-785. [CrossRef] [PubMed]

65. Ottery, F.D. Definition of standardized nutritional assessment and interventional pathways in oncology. Nutrition 1996, 12, S15-S19. [CrossRef]

66. Love, M.I.; Huber, W.; Anders, S. Moderated estimation of fold change and dispersion for RNA-seq data with DESeq2. Genome Biol. 2014, 15, 550. [CrossRef]

67. Stephens, N.A.; Gallagher, I.J.; Rooyackers, O.; Skipworth, R.J.; Tan, B.H.; Marstrand, T.; Ross, J.A.; Guttridge, D.C.; Lundell, L.; Fearon, K.C.; et al. Using transcriptomics to identify and validate novel biomarkers of human skeletal muscle cancer cachexia. Genome Med. 2010, 2, 1. [CrossRef]

68. Kupershmidt, I.; Su, Q.J.; Grewal, A.; Sundaresh, S.; Halperin, I.; Flynn, J.; Shekar, M.; Wang, H.; Park, J.; Cui, W.; et al. Ontology-based meta-analysis of global collections of high-throughput public data. PLoS ONE 2010, 5, e13066. [CrossRef] 